\title{
Influence of Melanin Content on Laser Doppler Flowmetry and Tissue Reflectance Oximetry Signal Formation
}

\author{
Viktor Dremin ${ }^{1,2^{*}}$, Nadezhda Golubova ${ }^{1}$, Elena Potapova ${ }^{1}$, and Andrey Dunaev ${ }^{1}$ \\ ${ }^{1}$ Research and Development Center of Biomedical Photonics, Orel State University, 95 Komsomolskaya str., \\ Orel 302026, Russia \\ ${ }^{2}$ College of Engineering and Physical Sciences, Aston University, Aston Triangle, Birmingham B4 7ET, UK \\ * e-mail: v.dremin1@aston.ac.uk
}

\begin{abstract}
The article presents the results of studies of the melanin effect on recorded signals in laser Doppler flowmetry (LDF) and tissue reflectance oximetry (TO). By investigating the properties of recorded microvascular blood flow and skin oxygenation signals, we have gained new knowledge about racerelated differences in the formation of these signals. The effect of the skin melanin content on the intensity of the recorded signals in the LDF and TO methods was evaluated, and the limitations in the use of these methods in representatives of different ethnic groups were shown. (C) 2021 Journal of Biomedical Photonics \& Engineering.
\end{abstract}

Keywords: laser Doppler flowmetry; blood perfusion; tissue reflectance oximetry; tissue oxygen saturation; melanin.

Paper \#3452 received 15 Aug 2021; revised manuscript received 5 Oct 2021; accepted for publication 11 Oct 2021; published online 5 Nov 2021. doi: 10.18287/JBPE21.07.040306.

\section{Introduction}

Disorders of the blood microcirculation system are diverse both in their pathogenesis and clinical manifestations, in this regard, the microhaemodynamic state assessment is quite relevant. This is especially important in cardiology, diabetology, oncology, as well as in violation of microcirculation in dermatology, dentistry, surgery, and resuscitation.

Currently, laser Doppler flowmetry (LDF) and laser Doppler imaging are widely used methods for in vivo evaluation of the state of microcirculatory blood flow [1-5]. Laser Doppler measures the total local microcirculatory blood perfusion. This technique is based on tissue sensing by laser light and analysis of the light scattered by the tissue. Light impinging on moving red blood cells undergoes a Doppler shift in frequency, while light hitting static objects remains unchanged. A photodetector ultimately registers the scattered radiation. This allows for a continuous measurement of tissue perfusion based on Doppler-shifted and non-shifted photons registered by the photodetector.

Tissue reflectance oximetry (TO) is a technique employed for the non-invasive in vivo monitoring of system microhaemodynamics [6]. The tissue reflectance oximetry method provides information about the tissue oxygen saturation $\left(\mathrm{S}_{\mathrm{t}} \mathrm{O}_{2}\right)$ of the examined biological tissue microcirculation and allows for calculation of the relative blood volume $\left(V_{b}\right)$ in the surface layers of soft tissues (skin, mucous membranes). This technology is based on the difference in the optical properties of oxygenated $\left(\mathrm{HbO}_{2}\right)$ and deoxygenated $(\mathrm{Hb})$ haemoglobin when irradiated with green and red light [7-9]. Furthermore, in conjunction with LDF and other optical methodologies, tissue oximetry is a powerful tool capable of providing non-invasive measurements of parameters such as oxygen consumption within a living tissue. Tissue oxygen saturation is defined as the percentage composition of oxyhaemoglobin in the sum of two haemoglobin fractions, oxyhaemoglobin and deoxyhaemoglobin [10]:

$$
S_{t} O_{2}=\frac{C_{\mathrm{HbO}_{2}}}{C_{\mathrm{HbO}_{2}}+C_{H b}} \times 100 \%
$$

where, if the molar concentration of $\mathrm{HbO}_{2}$ in the blood is designated as $\mathrm{C}_{\mathrm{HbO} 2}$ and the overall molar concentration of all the fractions of haemoglobin in the blood, including $\mathrm{HbO}_{2}$, is designated as the sum of $C_{\mathrm{HbO} 2}$ and $C_{H b}$, then the parameter $V_{b}$ can be calculated as follows:

$$
V_{b}=\frac{C_{\mathrm{HbO}_{2}}+C_{H b}}{C_{\mathrm{HbO}_{2}}+C_{H b}+C_{\text {other }}} \times 100 \%
$$


where $C_{\text {other }}$ is the molar concentration of all secondary cellular structures in the examined volume of tissue.

The value of the molar concentration is determined by the absorption by biological tissue of radiation at different wavelengths. On the basis of the well-known Beer-Lambert law and taking into account multicomponent biotissue, the absorption coefficient can be calculated using the following formula:

$$
\mu_{a}(\lambda)=\sum_{i} \varepsilon_{i}(\lambda) \cdot C_{i}
$$

where $\varepsilon_{i}(\lambda)$ is the molar extinction coefficient for the biochemical medium component $i$, while $C_{i}$ is the molar concentration of component $i$ in the tested region.

Taking into account certain restrictions, the molar concentrations of the haemoglobin fractions vital for the calculation of $\mathrm{S}_{\mathrm{t}} \mathrm{O}_{2}$ and $V_{b}$ can be calculated using the following system of linear equations [10-12]:

$$
\left\{\begin{array}{l}
\mu_{a}\left(\lambda_{1}\right)=\varepsilon_{H b}\left(\lambda_{1}\right) \cdot C_{H b}+\varepsilon_{H b O_{2}}\left(\lambda_{1}\right) \cdot C_{H b O_{2}}+\varepsilon_{\text {other }}\left(\lambda_{1}\right) \cdot C_{\text {other }} \\
\mu_{a}\left(\lambda_{2}\right)=\varepsilon_{H b}\left(\lambda_{2}\right) \cdot C_{H b}+\varepsilon_{H b O_{2}}\left(\lambda_{2}\right) \cdot C_{H b O_{2}}+\varepsilon_{\text {other }}\left(\lambda_{2}\right) \cdot C_{\text {other }}
\end{array}\right.
$$

where $\varepsilon_{\text {other }}\left(\lambda_{j}\right)$ is the sum molar extinction coefficient of light at different wavelengths by all other skin structures.

Human skin is a multicomponent turbid absorbing and scattering medium. Absorption of radiation is one of the characteristics of the interaction of light and skin, due to the presence of a large number of different chromophores in tissues [13]. However, such a chromophore as melanin has an individual pattern of absorption (see Fig. 1a), since its concentration can vary greatly from one person to another and depending on the place of measurement, which can lead to incorrect diagnostic results. The melanocytes located in the cell basal layer produce melanin and transfer it to neighbouring cells of the epidermis keratinocytes in order to protect their nucleus from UV radiation. Regardless of the ethnicity in the skin of each person is the same amount of melanocytes, but the amount of melanin, which these cells produce, is different. The amount of produced melanin determines the colour of the skin and its sensitivity to light. In people with different ethnic skin types, the concentration of melanin varies approximately in the range of $1.3-43 \%$. In lowpigmented skin, the melanin content is $1.3-1.6 \%$, $11-16 \%$ in middle-pigmented, $18-43 \%$ in blacks [13].

In this regard, in the methods of optical diagnostics, including LDF and TO, there is a problem of correct assessing of the microhaemodynamics state and determining the concentration of oxyhaemoglobin in people belonging to different ethnic groups, since the differences in the optical properties of the skin of these people are not taken into account. The work [14] presents studies of race-specific differences in the dynamics of blood flow and tissue oxygenation and shows the specificity of the difference in the phase coherence of LDF signals and tissue oximetry. This paper discusses the natural physiological difference in the recorded parameters of the cardiovascular dynamics between people of Sub-Saharan African descent and European descent. In the current work, we consider the effect of melanin as an optical filter, both in experimental studies on various ethnic skin types, and using computational modelling.

\section{Experimental System and Methodology}

Experimental studies were carried out using a multifunctional laser non-invasive certified diagnostic system "LAKK-M" (SPE “LAZMA" Ltd., Moscow, Russia), the appearance of which is presented in Fig. 1b [15-17]. The system is designed for research and diagnostics in various fields of biomedicine by simultaneously using the methods of pulse oximetry, laser Doppler flowmetry (LDF), tissue reflectance oximetry, and fluorescence spectroscopy. It allows one to study of a number of microcirculatory parameters, including perfusion (by LDF) and oxygen saturation $\left(\mathrm{S}_{\mathrm{t}} \mathrm{O}_{2}\right)$ of skin tissue (by TO). The device apparatus includes a fibre-optic system to allow for analysing at selected points on the body. The wavelength of the probe laser radiation in the LDF channel is $1064 \mathrm{~nm}$, in the TO channels $-530 \mathrm{~nm}$ and $630 \mathrm{~nm}$, accordingly. A multioptical fibre probe was used to delivery of radiation
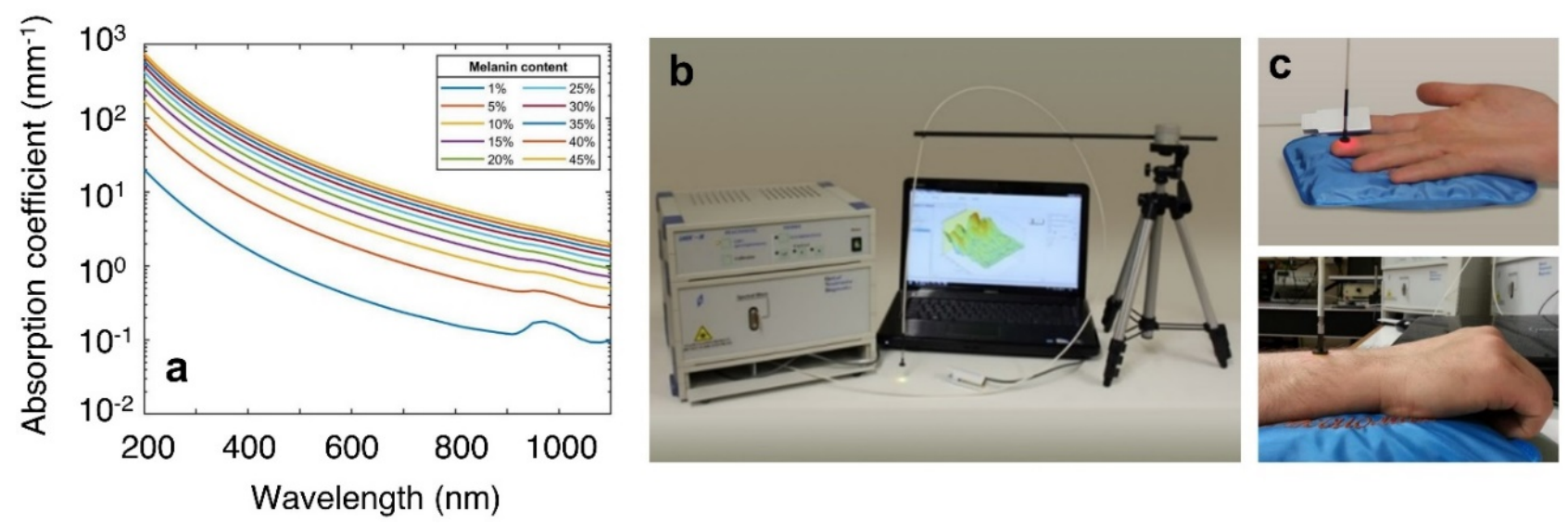

Fig. 1 (a) Absorption spectra of the epidermis with different melanin content [18]. (b) The multi-functional laser non-invasive diagnostic system "LAKK-M". (c) Measurements areas are the finger pad and the forearm. 
and registration of backreflected radiation from the tissue. The probing single-mode fibre of the LDF channel has a diameter of $6 \mu \mathrm{m}$ and the probing fibre of the TO channel $400 \mu \mathrm{m}$. All receiving fibres have a diameter of $400 \mu \mathrm{m}$. The numerical aperture of the fibres is 0.22 . The output power of LDF and TO lasers does not exceed $1.5 \mathrm{~mW}$ and $1 \mathrm{~mW}$, respectively.

We studied the effect of the skin melanin on the LDF and TO parameters as recorded on different ethnicities including Caucasian (type II of Fitzpatrick scale, 4 volunteers, totalling 92 records), Indian (type IV of Fitzpatrick scale [19], 1 volunteer, 30 records), Middle Eastern (type V of Fitzpatrick scale, 1 volunteer, 16 records) and African (type VI of Fitzpatrick scale, 3 volunteers, 4 records). The inclusion of a large number of measurements with Caucasian skin type allowed to demonstrate that even with high individual variability of the measured parameters, statistically significant differences remain in different ethnic skin types. Recordings on volunteers with Caucasian skin type were carried out during the calendar year, which allowed us to additionally take into account the seasonal effects of skin melanin.

The study was performed approximately at the same time of day (around 12:00) to avoid circadian rhythms influence on the blood circulation, at ambient room temperature $\left(21-22^{\circ} \mathrm{C}\right)$ in a sitting position after a $30 \mathrm{~min}$ rest period (allowed for acclimatization). The temperature of the volunteers was measured in the tested regions of the skin using an infrared clinical thermometer. All temperatures recorded were within the range of $34-36{ }^{\circ} \mathrm{C}$. Measurements were performed on the inner surface (palmar) of the right middle finger (slightly pigmented area for all volunteers), and on the skin of the forearm in the area with the differences in the melanin content (Fig. 1c). An equal number of records were recorded from each skin area. Recording of the signals lasted $3 \mathrm{~min}$.

\section{Results}

\subsection{Laser Doppler Flowmetry Measurements}

LDF signals were obtained for people with different ethnic skin types, typical graphs of which are shown in Figs. 2 (a, b).

The analysis of LDF signals within the groups was carried out using statistical mean values of blood perfusion, standard deviation, confidence interval, and coefficient of variation (see Fig. 2 (c, d) and Table 1). A statistically significant differences between the groups were confirmed by the Kruskal-Wallis test with the posthoc analysis $(p<0.05)$. Evaluating the results obtained in the area of the finger pad (Fig. 2c), it can be noted that the perfusion values are the same, which is probably due to approximately the same level of melanin content. Analysing the results in the forearm area, one can notice that in the group of volunteers with African skin type, there was a decrease in signal compared to other groups of volunteers (Fig. 2d).
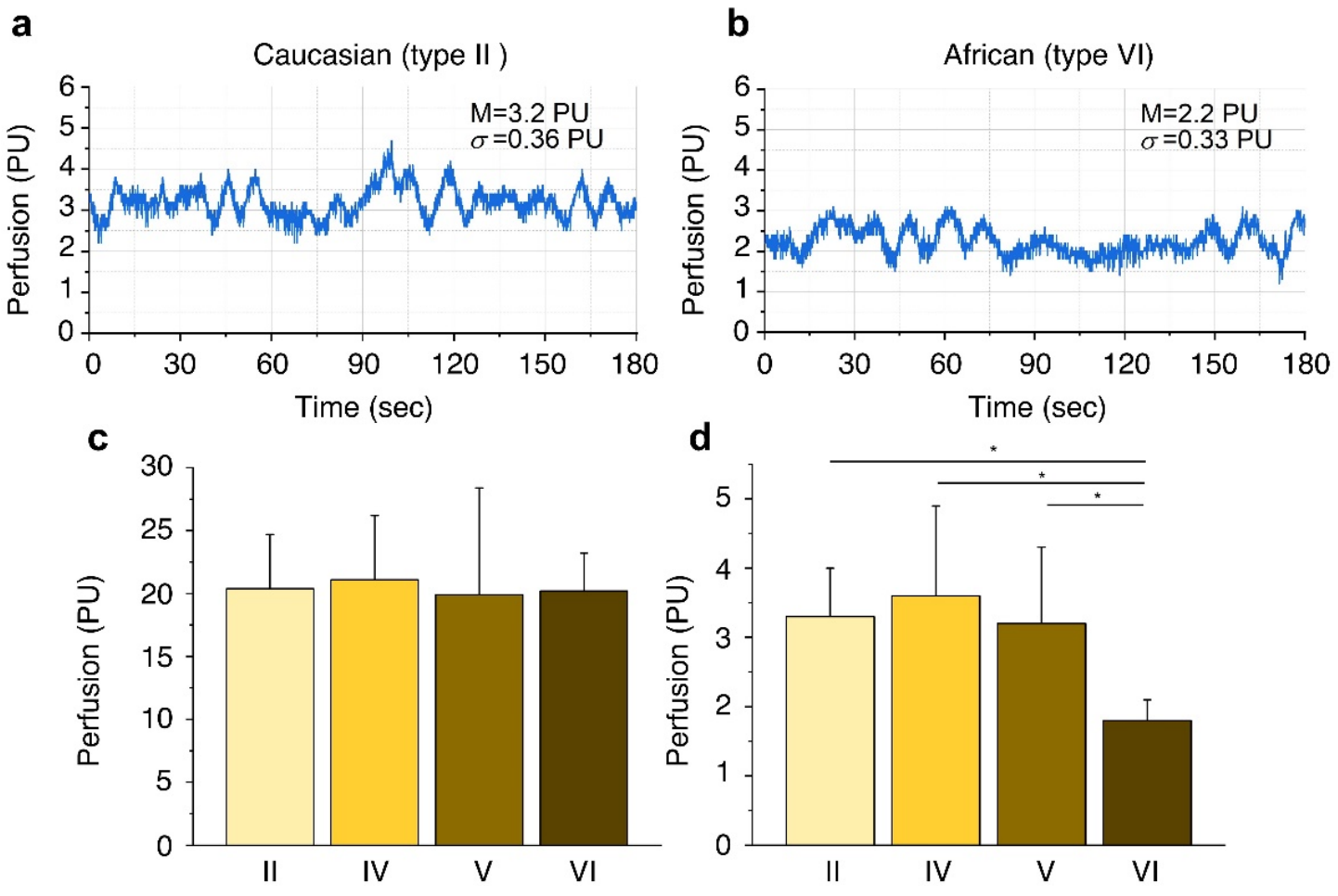

Fig. 2 Typical LDF signals for (a) Caucasian and (b) African skin types in area of the forearm. Average values and standard deviation of perfusion for different ethnic groups in the area of (c) the finger and (d) the forearm. II, IV, V, and VI are Fitzpatrick skin tone types. * Confirmed statistically significant differences $(p<0.05)$. 
Table 1 Confidence interval and coefficient of variation of the measured and calculated parameters within the groups.

\begin{tabular}{|c|c|c|c|c|c|c|c|}
\hline \multirow{2}{*}{ Statistic } & \multirow{2}{*}{$\begin{array}{l}\text { Fitzpatrick } \\
\text { scale }\end{array}$} & \multicolumn{3}{|c|}{ Finger } & \multicolumn{3}{|c|}{ Forearm } \\
\hline & & Perfusion & $\mathrm{St}_{\mathrm{t}} \mathrm{O}_{2}$ & $V_{b}$ & Perfusion & $\mathrm{St}_{\mathrm{t}} \mathrm{O}_{2}$ & $V_{b}$ \\
\hline \multirow{4}{*}{$\begin{array}{l}\text { Confidence } \\
\text { interval }\end{array}$} & II & $19.5-21.3$ & $74.2-78.2$ & $10.4-11.4$ & $3.1-3.5$ & $67.08-71.12$ & $5.57-6.23$ \\
\hline & IV & 18.9-23.3 & $72.3-75.3$ & $11.4-13.0$ & $3.0-4.2$ & $60.89 ; 65.11$ & $10.02-11.58$ \\
\hline & $\mathrm{V}$ & $14.6-25.2$ & $65.2-71.0$ & $10.8-12.3$ & $2.5-3.9$ & $53.72-58.08$ & $14.59-16.21$ \\
\hline & VI & $13.9-26.5$ & $52.6-85.2$ & $7.7-16.9$ & $1.2-2.4$ & $31.41 ; 40.59$ & $19.32-37.28$ \\
\hline \multirow{4}{*}{$\begin{array}{l}\text { Coefficient } \\
\text { of variation, } \\
\%\end{array}$} & II & 21 & 11 & 18 & 21 & 12 & 24 \\
\hline & IV & 24 & 5 & 15 & 36 & 8 & 17 \\
\hline & V & 43 & 7 & 10 & 34 & 6 & 8 \\
\hline & VI & 15 & 11 & 18 & 17 & 6 & 15 \\
\hline
\end{tabular}
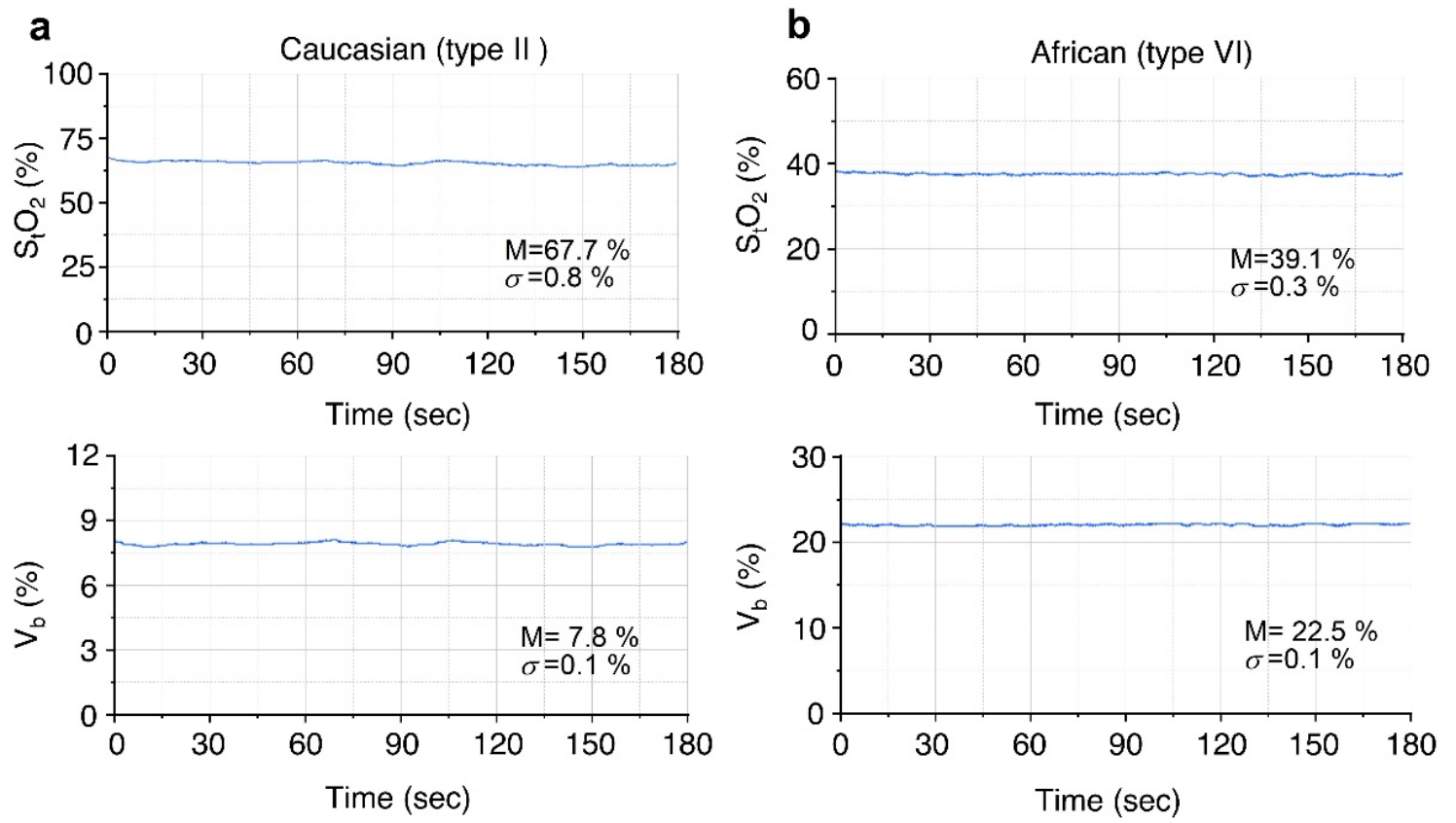

Fig. 3 Typical $\mathrm{S}_{\mathrm{t}} \mathrm{O}_{2}$ and $V_{b}$ signals recorded on the forearm for (a) European and (b) African skin types.

\subsection{Tissue Reflectance Oximetry Measurements}

Examples of typical $\mathrm{S}_{\mathrm{t}} \mathrm{O}_{2}$ and $V_{b}$ records for European and African skin types (area on the forearm) are shown in Figs. $3(\mathrm{a}, \mathrm{b})$, respectively. The tissue saturation parameter for the European skin type in this area of research is equal to the average, since normally this parameter is approximately within the range of $60-80 \%$. The obtained parameter represents tissue oxygenation and corresponds to the average between the oxygen saturation of the arterial (95-99\%) and venous (70-75\%) blood [20]. However, in the presented TO signals for African skin type, tissue saturation equals $39 \%$, which clearly demonstrates the inadequacy results for people with an increased content of skin melanin. The same applies to the $V_{b}$ parameter. Confirmation of the thesis that the differences are not due to ethnic physiological characteristics, but to the absorption capacity of melanin, is the different values of oxygenation in different areas of the skin for the same volunteer.

The obtained results registered in TO during the experiments were statistically processed according to the Kruskal-Wallis test (Fig. 4). As a result of the processing, a statistical difference was found in the calculated values obtained during the forearm measurements in ethnic groups with an increased melanin content compared to the type of white European skin type $(p<0.05)$. Thus, the measurements results are strongly dependent on the level of melanin present in the skin.

In the next Section, to describe and predict the effect of melanin on the intensity of the diffusely reflected radiation component, a Monte Carlo (MC) simulation was performed. 

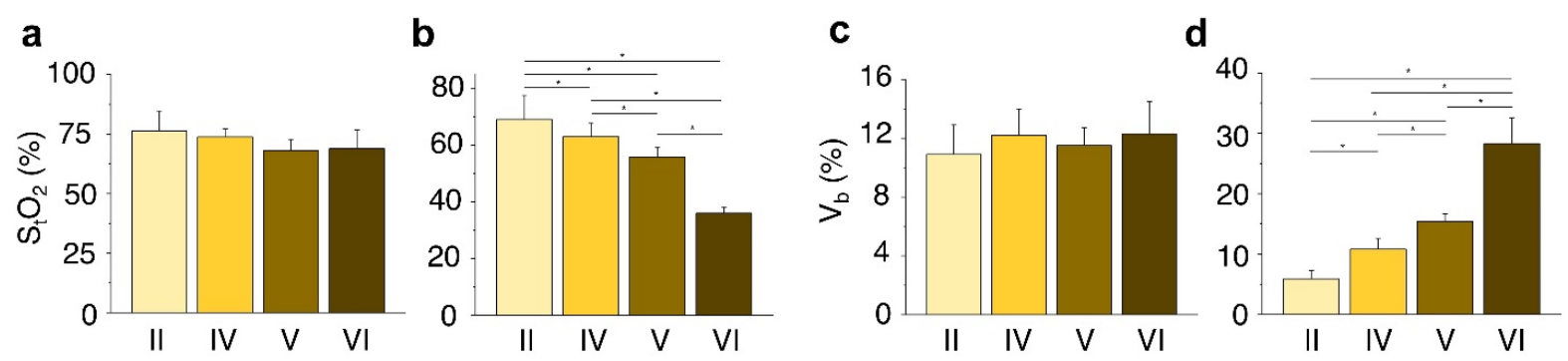

Fig. 4 The average values and standard deviation of $\mathrm{StO}_{2}$ and $V_{b}$ parameters for different ethnic groups in the $(\mathrm{a}, \mathrm{c})$ finger and (b,d) forearm area. II, IV, V, and VI are Fitzpatrick skin tone types. * Confirmed statistically significant differences $(p<0.05)$.

\subsection{Monte Carlo Simulation of Diffuse Reflectance Intensity}

$\mathrm{MC}$ simulation consists of a sequential generation of trajectories of so-called photon packets from the site of photons' entrance into the medium to the area where the photon leaves the. In this study, a GPU-accelerated MC distributed online computational platform that implements an object-oriented concept [21] was used for a routine simulation of diffuse reflectance intensity. A seven-layer skin model was used to calculate the diffuse reflectance intensity. The basics of the considered model have been previously described in Refs. [22, 23]. The absorption coefficient of each layer takes into account the blood volume fraction in various vascular beds, oxygen saturation, water and melanin content $[24,25]$. The melanin content varied in the range of $1-45 \%$.

Optical properties of tissues used in MC simulations is provided in Ref. [25]. MC simulations were performed for the actual probe geometry used in the experiment using the detected photon packets. The total diffuse reflectance intensity has been calculated as the normalized sum of statistical weights of the photon packets satisfying the detection condition according to the microscopic Beer-Lambert law as follows:

$$
I(\lambda)=\frac{1}{N_{p h} W_{0}} \sum_{j}^{N_{p h}} W_{j} \exp \left(-\sum_{i=1}^{K_{i}} \mu_{a i}(\lambda) l_{i}\right) .
$$

Here, $N_{p h}$ is the number of detected photon packets, $W_{0}$ is the initial statistical weight of a photon packet entering a scattering medium, $W_{j}$ is the final weight of the $j^{\text {th }}$ detected photon packet, $K_{i}$ represents the total number of scattering events experienced by the photon packet within the medium, $\mu_{a i}$ is the medium local absorption and $l_{i}$ is the path length of the photon packet at $i^{\text {th }}$ step.

The results of the MC simulations are shown in Fig. 5.

The results presented in Fig. 5 demonstrate the changes in the intensity of diffuse reflectance of human skin resulting from variations in melanin content. Reflectance intensity for wavelength $1064 \mathrm{~nm}$ decreases by $33 \%$ for highly pigmented skin, for wavelengths of $530 \mathrm{~nm}$ and $630 \mathrm{~nm}$, these values are $86 \%$ and $85 \%$, respectively.

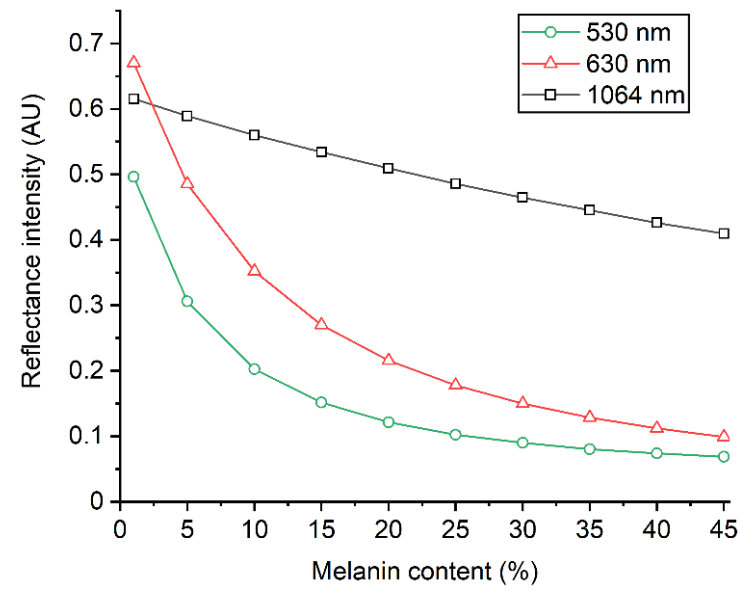

Fig. 5 The results of MC simulation of diffuse reflectance intensity while varying the melanin content in epidermis. The wavelengths of light used in the modelling are $530 \mathrm{~nm}, 630 \mathrm{~nm}$, and $1064 \mathrm{~nm}$.

\section{Discussion}

For many years of experience in using devices for assessing microcirculation by LDF and TO methods, most of the studied healthy volunteers and patients with various pathologies were with a slightly pigmented skin type. Most of the devices that implement these methods were probably calibrated without taking into account the data on the melanin content in the skin. Furthermore, diagnostic methods indicating the norm and pathology were developed in most cases for people with light skin.

Studies of the effect of the melanin pigment on the recorded optical signals by methods of optical noninvasive diagnostics show that this aspect is important to take into account when developing various types of devices. Most studies on the influence of skin type on recorded signals are devoted to photoplethysmography and pulse oximetry due to their widespread use in medical practice. It has been shown that a dark skin colour can affect the performance and accuracy of pulse oximeters from various manufacturers [26, 27]. For the correct interpretation of the diagnostic results, it is necessary to take into account the influence of skin type and wavelength on light reflection [28]. A device with an automatic adaptation module with an optimal choice of suitable lighting was proposed for different skin types [29]. In our early studies, we studied the effect of 
melanin on the attenuation of fluorescence intensity and proposed methods for assessing this effect [30, 31]. A group led by Prof. Aneta Stefanovska showed differences in cardiovascular and microvascular dynamic processes between black Africans and Europeans, using records obtained using NIRS, white light spectroscopy and LDF methods [14].

In the present paper, we study the effect of melanin on LDF and TO signals in both experimental studies and using modelling. It was found that the influence of melanin on the recorded signals strongly depends on the location of the recorded signal: statistically significant differences were found in the forearm area, which is due to the fact that in this area of research, differences in the influence of melanin are most pronounced. The results obtained demonstrate that the skin type on the Fitzpatrick scale significantly affects the measured parameters. In the area of strong pigmentation (forearm) for groups II, $\mathrm{IV}$, and $\mathrm{V}$, there were no statistically significant differences in the perfusion parameter. However, an increase in pigmentation to group VI (melanin content of more than $20 \%$ ) can lead to a decrease in perfusion by $45 \%$ compared to type II skin. $\mathrm{S}_{\mathrm{t}} \mathrm{O}_{2}$ and $V_{b}$ parameters are even more sensitive to the melanin content. A sequential increase in melanin from group II to IV, from IV to $\mathrm{V}$, and from $\mathrm{V}$ to $\mathrm{VI}$ causes a decrease in the $\mathrm{S}_{\mathrm{t}} \mathrm{O}_{2}$ parameter by 9,11 , and $36 \%$, respectively. At the same time, there is an increase in the $V_{b}$ parameter by 83,43 , and $84 \%$, respectively.

Since the development of wearable devices, including those implementing the LDF and TO methods [32, 33], which most often use this area for research, is gaining momentum in medical instrumentation, the presented results are important and should be taken into account.

Currently, various wavelengths are used in laser Doppler flowmetry devices. Moor Instruments Ltd. (Axminster, UK) and Oxford Optronix Ltd. (Oxford, UK) devices use a wavelength of $785 \mathrm{~nm}$, Perimed AB (Järfälla, Sweden) - 780 nm, , SPE "LAZMA" Ltd. (Moscow, Russia) - 1064 nm. As can be seen in Fig. 1a, as the wavelength increases, the absorption of the optical signal by melanin decreases. In this regard, a longer wavelength is considered to be more suitable for measurements on highly pigmented skin. The wavelengths used in the TO channel $(530 \mathrm{~nm}$ and $630 \mathrm{~nm}$ ) are strongly influenced by the absorption of melanin (Fig. 1a). In this sense, the intensity of backreflected radiation decreases in the forearm area with an increase in skin pigmentation, which ultimately affects the signal-to-noise ratio and the values of the calculated parameters. This is important to take into account when developing new wearable devices, and in the instructions for existing devices, it is necessary to specify restrictions related to skin colour.

The simulation results confirm the need to develop methods and algorithms for taking into account the effect of melanin on the results of optical measurements, in particular, approaches for personalized calibration of diagnostic systems. In this direction, the use of neural networks to restore the concentrations of various skin chromophores seems promising. In works [24, 34], the possibilities of such an approach were demonstrated, in which the melanin content was taken into account when determining the oxygenation of the skin by hyperspectral imaging.

It is important to note that our study has some limitations. We tested a small group of volunteers and only one type of diagnostic system. A further perspective of the study is the frequency analysis of the rhythmic components of skin blood flow at different concentrations of melanin and probing wavelengths (from 600 to $1100 \mathrm{~nm}$ ), as well as the expansion of the experimental data base.

\section{Conclusion}

By investigating the properties of recorded microvascular blood flow and skin oxygenation signals, we have gained new knowledge about race-related differences in the formation of these signals.

The experimental data and simulation results are a clear illustration of the fact that the levels of signals recorded in LDF and TO when probing the skin with the radiation of green, red and infrared sources for groups of people with different ethnic skin types is significantly different. And as a result, the calculated values (perfusion, $\mathrm{S}_{\mathrm{t}} \mathrm{O}_{2}, V_{b}$ ) are incorrect for ethnic skin types with an increased level of melanin, in contrast to the European type, for which the current instrumental implementation of the LDF and TO channels is calibrated.

Therefore, it can be concluded that it is necessary to take into account the individual optical parameters of the human skin. For example, in subjects with different levels of skin melanin, one should choose areas of investigation that will help avoid the effect of shielding optical signals from hyperpigmented skin (e.g., the ventral surface of the hand). The contribution to the total absorption of cutaneous melanin should be taken into account both when creating mathematical models for these diagnostic technologies, and for their instrumental implementations.

\section{Disclosures}

All authors declare that there is no conflict of interests in this paper.

\section{Acknowledgments}

The team of the R\&D Center of Biomedical Photonics (Orel, Russia) has been inspired by the works of Dr. Ekaterina Borisova for many years. We knew Dr. Borisova personally and collaborated with her on some projects. Our team expresses its deep condolences to her relatives and colleagues. We mourn the loss of an important scientist in the world of biophotonics.

VD acknowledges for personal support from the European Union's Horizon 2020 research and innovation programme under the Marie Sklodowska-Curie grant agreement No. 839888. 
The authors acknowledge the support of the grant of the President of the Russian Federation for state support of young Russian scientists No. MK-398.2021.4.

\section{References}

1. R. Bonner, R. Nossal, "Principles of laser-Doppler flowmetry," in Laser-Doppler blood flowmetry. Developments in Cardiovascular Medicine, A. P. Shepherd, P. A. Öberg (Eds.), Springer, Boston, 17-45 (1990). ISBN: 978-1-47572083-9.

2. V. Dremin, E. A. Zherebtsov, I. N. Makovik, I. O. Kozlov, V. V. Sidorov, A. I. Krupatkin, A. V. Dunaev, I. E. Rafailov, K. S. Litvinova, S. G. Sokolovski, and E. U. Rafailov, "Laser Doppler flowmetry in blood and lymph monitoring, technical aspects and analysis," Proceedings of SPIE 10063, 1006303 (2017).

3. A. I. Zherebtsova, V. V. Dremin, I. N. Makovik, E. A. Zherebtsov, A. V. Dunaev, A. Goltsov, S. G. Sokolovski, and E. U. Rafailov, "Multimodal optical diagnostics of the microhaemodynamics in upper and lower limbs," Frontiers in Physiology 10, 416 (2018).

4. I. Makovik, A. V. Dunaev, V. V. Dremin, A. I. Krupatkin, V. V. Sidorov, L. S. Khakhicheva, V. F. Muradyan, O. V. Pilipenko, I. E. Rafailov, and K. S. Litvinova, "Detection of angiospastic disorders in the microcirculatory bed using laser diagnostics technologies," Journal of Innovative Optical Health Sciences 11(01), 1750016 (2018).

5. K. Kandurova, V. Dremin, E. Zherebtsov, E. Potapova, A. Alyanov, A. Mamoshin, Y. Ivanov, A. Borsukov, and A. Dunaev, "Fiber-optic system for intraoperative study of abdominal organs during minimally invasive surgical interventions," Applied Sciences 9(2), 217 (2019).

6. H. Liu, M. Kohl-Bareis, and X. Huang, "Design of a tissue oxygenation monitor and verification on human skin," Proceedings of SPIE-OSA Biomedical Optics 8087, 80871Y (2011).

7. C. Casavola, L. A. Paunescu, S. Fantini, M. A. Franceschini, P. M. Lugara, and E. Gratton, “Application of nearinfrared tissue oxymetry to the diagnosis of peripheral vascular disease," Clinical Hemorheology and Microcirculation 21(3-4), 389-393 (1999).

8. M. Wallace, A. Wax, D. N. Roberts, and R. N. Graf, "Reflectance spectroscopy," Gastrointestinal endoscopy clinics of North America 19(2), 233-242 (2009).

9. E. Potapova, V. V. Dremin, E. A. Zherebtsov, I. N. Makovik, A. I. Zherebtsova, A. V. Dunaev, K. V. Podmasteryev, V. V. Sidorov, A. I. Krupatkin, L. S. Khakhicheva, and V. F. Muradyan, "Evaluation of microcirculatory disturbances in patients with rheumatic diseases by the method of diffuse reflectance spectroscopy," Human Physiology 43(2), $222-228$ (2017).

10. A. V. Dunaev, V. V. Sidorov, A. I. Krupatkin, I. E. Rafailov, S. G. Palmer, N. A. Stewart, S. G. Sokolovski, and E. U. Rafailov, "Investigating tissue respiration and skin microhaemocirculation under adaptive changes and the synchronization of blood flow and oxygen saturation rhythms," Physiological Measurement 35(4), 607-621 (2014).

11. O. Khalil, "Metabolites, noninvasive optical measurements," in Wiley Encyclopedia of Biomedical Engineering, Wiley, New York (2006).

12. H. Heusmann, J. Koelzer, and G. Mitic, "Characterization of female breasts in vivo by time-resolved and spectroscopic measurements in the near infrared spectroscopy," Journal of Biomedical Optics 1(4), 425-434 (1996).

13. S. Jacques, "Optical properties of biological tissues: A review," Physics in Medicine \& Biology 58(11), 37-61 (2013).

14. Y. Abdulhameed, P. McClintock, and A. Stefanovska, "Race-specific differences in the phase coherence between blood flow and oxygenation: A simultaneous NIRS, white light spectroscopy and LDF study," Journal of Biophotonics 13(4), e201960131 (2020).

15. K. Litvinova, I. E.Rafailov, A. V. Dunaev, S. G. Sokolovski, and E. U. Rafailov, "Non-invasive biomedical research and diagnostics enabled by innovative compact lasers," Progress in Quantum Electronics 56, 1-14 (2017).

16. D. Rogatkin, L. G. Lapaeva, E. N. Petritskaya, V. V. Sidorov, and V. I. Shumskiy, "Multifunctional laser noninvasive spectroscopic system for medical diagnostics and some metrological provisions for that," Proceedings of SPIE-OSA Biomedical Optics 7368, 73681Y (2009).

17. D. Rogatkin, S. G. Sokolovski, K. A. Fedorova, N. A. Stewart, V. V. Sidorov, and E. U. Rafailov, "Basic principles of design and functioning of multifunctional laser diagnostic system for non-invasive medical spectrophotometry," Proceedings of SPIE 7890, 78901H (2011).

18. S. Jacques, D. McAuliffe, "The melanosome: threshold temperature for explosive vaporization and internal absorption coefficient during pulsed laser irradiation," Photochemistry and Photobiology 53(6), 769-775 (1991).

19. T. Fitzpatrick, "The validity and practicality of sun-reactive skin types I through VI," Archives of Dermatology 124(6), 869-871 (1988).

20. K. Akons, E. Dann, and D. Yelin, "Measuring blood oxygen saturation along a capillary vessel in human," Biomedical Optics Express 8(11), 5342-5348 (2017).

21. A. Doronin, I. Meglinski, “Online object oriented Monte Carlo computational tool for the needs of biomedical optics,” Biomedical Optics Express 2(9), 2461-2469 (2011). 
22. G. Petrov, A. Doronin, H. T. Whelan, I. Meglinski, and V. V. Yakovlev, "Human tissue color as viewed in high dynamic range optical spectral transmission measurements," Biomedical Optics Express 3(9), 2154-2161 (2012).

23. I. Meglinski, A. Doronin, A. N. Bashkatov, E. Genina, and V. V. Tuchin, "Dermal component-based optical modeling ofskin translucency: Impact on skin color," in Computational Biophysics of the Skin, B. Querleux (Ed.), Pan Stanford Publishing Ltd., 25-62 (2014).

24. E. Zherebtsov, V. Dremin, A. Popov, A. Doronin, D. Kurakina, M. Kirillin, I. Meglinski, and A. Bykov, "Hyperspectral imaging of human skin aided by artificial neural networks," Biomedical Optics Express 10(7), 35453559 (2019).

25. V. Dremin, E. Zherebtsov, A. Bykov, A. Popov, A. Doronin, and I. Meglinski, "Influence of blood pulsation on diagnostic volume in pulse oximetry and photoplethysmography measurements," Applied Optics 58(34), 9398-9405 (2019).

26. A. Ries, L. Prewitt, and J. Johnson, "Skin color and ear oximetry," Chest 96(2), 287-290 (1989).

27. P. Bickler, J. Feiner, and J. Severinghaus, "Effects of skin pigmentation on pulse oximeter accuracy at low saturation," Anesthesiology 102(4), 715-719 (2005).

28. B. Fallow, T. Tarumi, and H. Tanaka, "Influence of skin type and wavelength on light wave reflectance," Journal of Clinical Monitoring and Computing 27(3), 313-317 (2013).

29. L. Yan, S. Hu, A. Alzahrani, S. Alharbi, and P. Blanos, "A multi-wavelength opto-electronic patch sensor to effectively detect physiological changes against human skin types," Biosensors 7(2), 22 (2017).

30. A. Dunaev, V. V. Dremin, E. A. Zherebtsov, I. E. Rafailov, K. S. Litvinova, S. G. Palmer, N. A. Stewart, S. G. Sokolovski, and E. U. Rafailov, "Individual variability analysis of fluorescence parameters measured in skin with different levels of nutritive blood flow," Medical Engineering \& Physics 37(6), 574-583 (2015).

31. V. Dremin, A. Dunaev, "How the melanin concentration in the skin affects the fluorescence-spectroscopy signal formation," Journal of Optical Technology 83(1), 43-48 (2016).

32. M. Saha, V. Dremin, I. Rafailov, A. Dunaev, S. Sokolovski, and E. Rafailov, "Wearable laser Doppler flowmetry sensor: a feasibility study with smoker and non-smoker volunteers," Biosensors 10(12), 201 (2020).

33. A. Fedorovich, Y. L. Loktionova, E. V. Zharkikh, M. A. Mikhailova, J. A. Popova, A. V. Suvorov, and E. A. Zherebtsov, "Body position affects capillary blood flow regulation measured with wearable blood flow sensors," Diagnostics 11(3), 436 (2021).

34. V. Dremin, Z. Marcinkevics, E. Zherebtsov, A. Popov, A. Grabovskis, H. Kronberga, K. Geldnere, A. Doronin, I. Meglinski, and A. Bykov, "Skin complications of diabetes mellitus revealed by polarized hyperspectral imaging and machine learning," IEEE Transactions on Medical Imaging 40(4), 1207-1216 (2021). 